\title{
Unifying Life History Analyses for Inference of Fitness and Population Growth
}

\author{
Ruth G. Shaw \\ Department of Ecology, Evolution, and Behavior, Minnesota Center for Community \\ Genetics, University of Minnesota, St. Paul, Minnesota 55108 \\ rshaw@superb.ecology .umn.edu \\ Charles J. Geyer \\ School of Statistics, University of Minnesota, Minneapolis, Minnesota 55455 \\ charlie@stat.umn.edu \\ Stuart Wagenius
}

Institute for Plant Conservation Biology, Chicago Botanic Garden, Glencoe, Illinois 60022

\author{
swagenius@chicagobotanic.org \\ Helen H. Hangelbroek \\ Department of Ecology, Evolution, and Behavior, University of Minnesota, St. Paul, \\ Minnesota 55108 \\ helen_hangelbroek@hotmail.com \\ and \\ Julie R. Etterson
}

Biology Department, University of Minnesota-Duluth, Duluth, Minnesota 55812

jetterso@d.umn.edu 
Received __ ; accepted

Prepared with AASTEX - Type of submission: Article 


\begin{abstract}
The lifetime fitnesses of individuals comprising a population determine its numerical dynamics, and genetic variation in fitness results in evolutionary change. The dual importance of individual fitness is well understood, but empirical fitness records generally generally violate the assumptions of standard statistical approaches. This problem has plagued comprehensive study of fitness and impeded empirical study of the link between numerical and genetic dynamics of populations. Recently developed aster models address this problem by explicitly modeling the dependence of later expressed components of fitness (e.g. fecundity) on those expressed earlier (e.g. survival to reproduce). Moreover, aster models employ different sampling distributions for components of fitness, as appropriate (e.g. binomial for survival over a given interval and Poisson for fecundity). The analysis is conducted by maximum likelihood, and the resulting compound distributions for lifetime fitness closely approximate the observed data. We illustrate the breadth of aster's utility with three examples demonstrating estimation of the finite rate of increase, comparison of mean fitness among genotypic groups, and phenotypic selection analysis. Aster models offer a unified approach to address the breadth of questions in evolution and ecology for which life history data are gathered.
\end{abstract}

Subject headings: Chamaecrista fasciculata, community genetics, demography, Echinacea angustifolia, fitness components, Uroleucon rudbeckiae 
The fitness of an individual is well understood as its contribution, in offspring, to the population. Fitness has both evolutionary significance, as an individual's contribution to a population's subsequent genetic composition, and ecological importance, as an individual's numerical contribution to a population's growth. The simplicity of these closely linked ideas belies serious complications that arise in empirical studies. Lifetime fitness comprises multiple components of fitness expressed over one to many intervals. As a result, the distribution of fitness, even for a synchronized cohort in the absence of systematic sources of variation, is typically multimodal and highly skewed in shape and thus corresponds to no known parametric distribution. This problem has long been acknowledged (Mitchell-Olds and Shaw 1987; Stanton and Thiede 2005), yet to date there is no single, rigorously justified approach for jointly analyzing components of fitness measured sequentially throughout the lives of individuals. This limitation severely undermines efforts to link ecological and evolutionary inference.

Here we present applications of a new statistical approach, aster, for analyzing life-history data with the goal of making inferences about lifetime fitness or population growth. Aster modeling generates the overall likelihood for a set of components of fitness expressed through the lives of individuals. Within a single analysis, aster permits different fitness components to be modeled with different statistical distributions, as appropriate. It also accounts for the dependence of fitness components expressed later in the life-span on those expressed earlier. The statistical theory for aster models is presented in Geyer et al. (2007). Here, we first review the limitations of previous approaches to analysis of life-histories. Second, we describe aster models. Finally, we present three empirical examples to illustrate the utility of aster modeling as a comprehensive approach to analysis of life-history data. 


\section{The problem and previous efforts to address it}

Individual fitness realized over a lifespan typically does not conform to any well known distribution that is amenable to parametric statistical analysis. In contrast, individual components of fitness, such as survival to age $x$, reproduction at that age, and the number of young produced by a reproductive individual of that age, generally conform much more closely to simple parametric distributions. For this reason, components of fitness are sometimes analyzed separately to obviate the problem of the distribution of lifetime fitness. For example, in a study of genetic variation in response to conspecific density of a population of Salvia lyrata, Shaw (1986) provided separate analyses of two components of fitness, survival over two time intervals and size of the survivors, as a proxy for future reproductive capacity in this perennial plant. Implicitly, this approach considers size, or in other cases fecundity, conditional on survival. Though the statistical assumptions underlying the analyses tend to be satisfied, it offers no way to combine the analyses to yield inferences about overall fitness.

A common method for analyzing fitness as survival and reproduction jointly is to use fecundity as the index of fitness, assigning values of zero for fecundity of individuals that died prior to reproduction. When observations are available for replicate individuals, a variant of this method is to use as the measure of fitness the product of the proportion surviving and the mean fecundity of survivors (e.g. Belaoussoff and Shore 1995; Galloway and Etterson 2007). In both cases, the resulting distribution is actually a mixture of underlying discrete and (quasi)continuous distributions, yet analyses have generally treated it as a single, continuous response, despite its skewness and multimodality, such that no transformation yields a distribution suitable for parametric statistical analysis. Authors frequently remark on the awkwardness of these distributions in their studies (e.g. Etterson 2004), but rarely publish fitness distributions. Antonovics and Ellstrand (1984), however, 
presented the extremely skewed distribution of lifetime reproductive output (their Fig. 2)

76 from their experimental studies of frequency-dependent selection in the perennial grass, Anthoxanthum odoratum. Finding no transformation that yielded a normal distribution suitable for analysis of variance, they assessed the robustness of their inferences by applying three distinct analyses (categorical analysis of discrete fecundity classes, ANOVA of means, and nonparametric analysis). In this study, results of the three analyses were largely consistent, but, in general, results are likely to differ.

Others have noted the importance of complete accounting of life-history in inferring fitness or population growth rate, as well as evaluation of its sampling variation, and have presented methods to accomplish this. Caswell (2001) and Morris and Doak (2002) explain how to obtain population projection matrices from life-history records and, from them, to estimate population growth rate. They also describe methods for evaluating its sampling variation and acknowledge statistically problematic aspects of these methods. Specifically, Caswell (2001) notes (p.304) that the delta method and other series approximations assume both that variances of the elements of a population projection matrix are small and that the population growth rate is normally distributed. It is often further assumed that all the parameters are independent (Caswell (2001), p.302). These assumptions are likely to be violated in many cases. To avoid these assumptions, Caswell (2001) recommended resampling approaches, first applied in this context by Lenski and Service (1982), who emphasized that the complete life-history record of each individual is the unit of observation.

Recent efforts to evaluate the nature of selection have likewise taken a comprehensive demographic approach. McGraw and Caswell (1996) considered individual life-histories but chose the maximum eigenvalue of an individual's Leslie matrix $(\lambda)$ as its fitness measure. They regressed $\lambda$ on the fitness components, age at reproduction and lifetime reproductive output to estimate selection on them, but noted violation of the assumption of normality 
of residuals required for statistical testing. Van Tienderen (2000) advocated an alternative approach involving evaluation of the relationships between each component of fitness and the phenotypic traits of interest via separate multiple regression analyses to obtain the selection gradients in different episodes of selection (Lande and Arnold 1983). These selection gradients are then weighted by the elasticities (Caswell 2001) of each component of fitness obtained from analysis of the appropriate population projection matrix. Using this method, Coulson et al. (2003) also noted violation of the usual distributional assumption. Moreover, because the method combines results from multiple analyses, it does not fully account for sampling variation. Beyond these approaches, methods targeting the problem of "zero-inflated" data (i.e. many observations of zero distorting a distribution) have also been proposed (Cheng et al. 2000; Dagne 2004; Martin, et al. 2005). However, like the other methods, this method does not generalize readily for inference in the wide range of contexts that life-history data can, in principle, address.

\section{Inference of individual fitness with aster}

We present aster models (Geyer et al. 2007) for analysis of life-history records as a general, statistically sound approach to address diverse questions in evolution and ecology. As noted above, two standard properties of life-history data are central to the statistical challenges that aster addresses. First, the expression of an individual's life-history at one stage depends on its status at earlier stages. For example, observation of an individual's fecundity at one stage is contingent on its survival to that stage. Second, no single parametric distribution is generally suitable for modeling all components of fitness, e.g. survival and fecundity. The aster approach jointly models the components of fitness using distributions suitable for each and explicitly taking into account the inherent dependence of each stage on previous stages. We represent the life-history and, in particular, 
the dependence of one life-history component on another, with graphical models as in Fig. 1 with arrows pointing from a predecessor variable to its successor.

EDITOR: PLACE FIGURE 1 HERE.

The theory underlying the aster approach requires modeling the conditional distribution of each variable given its predecessor variable as an exponential family of distributions (Lehmann and Casella 1998; Barndorff-Nielsen 1978; Geyer et al. 2007) with the predecessor variable providing the sample size for its successors. This requirement retains considerable flexibility, because many well-known distributions are exponential families, including Bernoulli, Poisson, geometric, normal, and negative binomial (Mood, et al. 1974, p. 312 ff.). When questions arise about the applicability of aster models, some diagnostic tools are available, as demonstrated in our Example 3.

A predecessor variable $n$ must be nonnegative integer valued. If $n>0$, then the successor is the sum of $n$ independent and identically distributed variables having the named distribution. If $n=0$, then the successor is zero. This accommodates much of the dependence in life history data. In a graph like Fig. 1A, where each of the variables $\mathbf{S} x$ models survival (zero-or-one with one indicating alive), a dead individual stays dead and does not reproduce.

Aster is a general approach, suited to analyzing complicated life-histories (e.g. Fig. 1). Approaches commonly used for particular data structures are special cases of aster. The simplest possible aster models have graphs with only one arrow per individual $1 \rightarrow X$. If $X$ is normal, this is a linear model (LM) as in multiple regression or analysis of variance. If $X$ is Bernoulli or Poisson, this is a generalized linear model (GLM) as in logistic or Poisson regression (McCullagh and Nelder 1989). The next simplest models have graphs $1 \rightarrow X \rightarrow Y$ with $X$ Bernoulli and $Y$ zero-truncated Poisson (like Fig. 1D); here the 

$\mu=E(X)$ whereas the canonical parameter is $\theta=\log (\mu)$.

In all aster models, a transition between life-history stages, i. e. each arrow in the graph, corresponds to the conditional distribution of one fitness component, which contributes one term to the log likelihood

$$
l(\theta)=\sum_{j}\left[x_{j} \theta_{j}-x_{p(j)} c_{j}\left(\theta_{j}\right)\right]
$$

where $x_{j}$ is the canonical statistic and $\theta_{j}$ the canonical parameter for the $j$-th conditional distribution and $x_{p(j)}$ is the predecessor of $x_{j}$. Each term of (1) has exponential family form, but the sum does not. It can, however, be put in exponential family form

$$
l(\varphi)=\left[\sum_{j} x_{j} \varphi_{j}\right]-c(\varphi)
$$

by a change of parameter. Either (1) or (2) is a log likelihood for the full model with one parameter per variable, and the canonical statistic vector $x$ is the same for both, but the linearly modeled canonical parameters, $\theta$ and $\varphi$, differ. To distinguish the two canonical parameter vectors, we call $\theta$ conditional and $\varphi$ unconditional.

Unconditional aster models are submodels of the full model determined by the change of parameter $\varphi=M \beta$. The submodel is also an exponential family with log likelihood

$$
l(\beta)=\left[\sum_{k} y_{k} \beta_{k}\right]-d(\beta)
$$


where $y=M^{T} x$. The matrix $M$ is called the model or design matrix. For this submodel $y$ is the canonical statistic and $\beta$ is the canonical parameter whose maximum likelihood estimate (MLE) solves the equations

$$
y_{k}=E_{\beta}\left(Y_{k}\right)
$$

where $t_{j k}$ are the components of $T$, but it has no simple properties. The MLE is not a sufficient statistic and has no monotone relationship with expectations.

Either kind of aster model (conditional or unconditional) is a model for the joint distribution of all the data. Whereas either may be useful for some particular data, only unconditional models have simple interpretations in terms of unconditional mean values (of the canonical statistics), like those familiar from LM and GLM. We recommend them because, when lifetime fitness is a canonical statistic, these tests and confidence intervals directly address fitness. The unconditional parameterization is not readily understood intuitively because terms in $\beta$ that nominally refer to a single component of fitness (affect 
176

its $\varphi_{i}$ only) directly influence the unconditional expectation of overall fitness by affecting not only the distribution of the specified component, but also the distributions of its predecessor, predecessor of predecessor, etc. Consequently, it is difficult (but not impossible, see our Example 2), to see the role played by a single component of fitness. This is an unavoidable consequence of being able to address overall fitness.

We demonstrate the value and versatility of the aster approach with three examples. In the first, we illustrate inference of population growth rate. We consider a small dataset that Lenski and Service (1982) used to demonstrate their nonparametric method for inferring population growth rate from a set of individual life-histories of the aphid, Uroleucon rudbeckiae. In this case, we illustrate the use of a conditional model, though either form of model could be used. In our second example, we apply aster to compare mean fitness among groups. Specifically, we quantify effects of inbreeding on fitness of Echinacea angustifolia, a long-lived plant, showing confidence intervals for mean fitness (Fig. 2). In the last example, we reanalyze data of Etterson (2004) to evaluate phenotypic selection on the annual legume, Chamaecrista fasciculata, to estimate the fitness surface in relation to phenotypic traits. In this case, we show how much simpler aster analysis is when fitness is a canonical statistic of an unconditional model and also how to proceed when, due to the experimental design, it is not. A contributed package "aster" for the R statistical language (R Development Core Team 2006) does all calculations related to aster models, contains the datasets for our examples, and is freely available (http://www.r-project.org). Two technical reports (Shaw, et al. 2007a,b) give more extensive analyses, which are reproducible (see Chapter 1 of Shaw, et al. 2007a). 
Lenski and Service (1982) recognized the need for a valid statistical approach to inferring rates of population growth $(\lambda)$ from life-history records via the stable age equation (Fisher 1930). They emphasized the importance of accounting for individual variation in survivorship and fecundity and of treating the full life-history record of an individual as the unit of observation. Lenski and Service (1982) presented a nonparametric approach that resamples complete records of individual life histories via the jackknife procedure. Using the properties of the jackknife, they showed how to obtain estimates and sampling variances of $\lambda$. They illustrated the approach with a small dataset sampled from the aphid, Uroleucon rudbeckiae. The survival and fecundity in each of fourteen age intervals were recorded for 18 individuals in a cohort (see Fig. 1A), and these data served as the basis for estimating $\lambda$ and its sampling variance.

Applying aster to these data, we modeled the binomial parameter governing survival probability, $\operatorname{logit}\left(\sigma_{x}\right)$, as a quadratic function of age, $x$. Survivorship declined significantly with age $(P=0.001)$, significantly $(P=0.028)$ nonlinearly. Expected fecundities, $\beta_{x}$, modeled according to a Poisson distribution, were estimated for each age class, $x$, given survival to that age.

Interest focuses primarily on estimating $\lambda$, but also on its sampling variance, as noted by Alvarez-Buylla and Slatkin (1994), because of its importance in assessing whether a population is growing or declining. The stable age equation implicitly defines $\lambda$ as a nonlinear function of the unconditional expectations $\mu_{x}=\sigma_{x} \beta_{x}$, which are estimated by aster; from these, $\lambda$ is determined by solving the stable age equation, and standard errors are obtained using the delta method (Shaw, et al. 2007a, give details). From these data, we estimated $\lambda=1.677$ with a standard error of 0.056 . Our estimate agrees closely with that of Lenski and Service (1982) (1.688), and 95\% confidence intervals are also similar (aster: 
1.57, 1.79; jackknife: 1.52, 1.85). We emphasize, however, that the aster approach can be

\section{Example 2: Comparison of fitness among groups}

In this example, we illustrate use of aster models to compare mean fitnesses of groups. Specifically, we investigate how relatedness of parents affects progeny fitness in a perennial plant, Echinacea angustifolia (narrow-leaved purple coneflower), a widespread species in the N. American prairie and Great Plains. Following the conversion of land to agriculture and urbanization that began about a century ago, the formerly extensive populations now persist in typically small patches of remnant prairie. The plant is self-incompatible, and Wagenius (2000) detected no deviation from random mating in a large population in western Minnesota. In the context of fragmented habitat, matings between close relatives in the same remnant, and perhaps also long distance matings, may have become more common.

To evaluate the effects of different mating regimes on the fitness of the progeny, formal crosses were conducted between pairs of plants a) from different remnants, b) chosen at random from the same remnant, and c) known to share maternal parent. The parental plants had been growing for 3-4 years in randomized arrays in a common experimental field. From the resulting seeds, 557 seedlings were germinated. After three months in a growth chamber, the surviving 508 individuals were transplanted back into the same experimental field. Survival of each seedling was assessed in the growth chamber on three dates and, after transplanting into the field, annually, 2001-2005. The number of rosettes (basal leaf clusters, 1-7) per plant was also counted annually 2003-2005. Here, individual size is considered a component of fitness during the juvenile period; the typically strong positive relationship between size and eventual fecundity justifies this here, as elsewhere. 
Mortality of many plants $(\sim 30 \%)$ resulted in a distribution of rosette count in 2005

having many zeros. We modeled survival through each of eight observation intervals as Bernoulli, conditional on surviving through the preceding stage; we modeled rosette count in each of three field seasons, given survival to that season, as zero-truncated Poisson (Fig. 1B). To account for spatial and temporal heterogeneity, we also included in the models as fixed effects a) year of crossing (1999 or 2000), b) planting tray during the period in the growth chamber, c) spatial location (row and position within row) in the field.

Our primary focus is on evaluating the effects of mating treatments on overall progeny fitness, taken as expected rosette count in 2005 for a seed obtained in 2001. In addition, we investigated the timing and duration of the effects of mating treatment on fitness. These effects could be slight during the early stages but, cumulatively, could strongly influence overall fitness. Alternatively, it may be that the effects of mating treatment at the early stages largely account for their overall effects on fitness. These scenarios differ in their implications concerning the inbreeding load expected in standing populations (Husband and Schemske 1996). To evaluate these scenarios, we developed four aster models, named "chamber," "field," "sub," and "super." Each was a joint aster analysis of all 11 fitness components (survival over eight intervals, rosette count at three times). The "field" model, corresponding to the first scenario, includes explicit mating treatment effects only on the final rosette count (variable r05 in Fig. 1B), but because of the unconditional parameterization of aster models, these effects propagate back to earlier stages as well. The "chamber" model, referring to the second scenario, includes explicit mating treatment effects only on the final survival before transplanting (variable lds3 in Fig. 1B), but, again, these effects propagate back through the two preceding bouts of survival. The remaining two models are required to test the above scenarios of timing of effects; the "sub" model is the greatest common submodel of "chamber" and "field," and the "super" model is their least common supermodel (i. e. "sub" includes no effects of mating treatment on any aspect 
of fitness, whereas "super" includes separate effects of mating treatment on survival up to

\section{EDITOR: PLACE FIGURE 2 HERE.}

Because of the propagation of effects back to earlier stages, the effects of mating treatment on r05 in the "field" model directly account for expression of fitness at all earlier stages. Thus, this analysis suffices for inferring the overall effects of mating treatment on fitness.

Our further investigation of the timing and duration of these effects detects differences among mating treatments in survival up to transplanting (comparison of "sub" and "chamber" models $P=0.012$ ). Beyond this, the comparison of the "chamber" and "field" models with the "super" model shows that the "field" model accounts well for differences in expressed fitness; "super" fits no better than "field" $(P=0.34)$ but does fit better than "chamber" $\left(P=3.1 \times 10^{-4}\right)$. The terms in the "super" model that quantify the effect of mating treatment on survival up to transplanting are not needed to fit the data, because the back propagation of effects subsumes the effects of mating treatment in the growth chamber. This does not mean there are no effects of mating treatment on fitness before transplanting. The comparison of "sub" and "chamber" confirms they exist, and Fig. 2 clearly shows them. The fitness disadvantage of progeny resulting from sib-mating relative to the other treatments is clear in the $7 \%-10 \%$ reduced survival up to the time of transplanting but the overall fitness disadvantage of inbreds is considerably greater (Fig. 2A). 


\section{Example 3: Phenotypic selection analysis}

Lande and Arnold (1983) proposed multiple linear and quadratic regression of fitness

on a set of quantitative traits as a method for quantifying natural selection directly on each trait. In practice, these analyses have generally employed measures of components of fitness as the response variable, rather than overall fitness (e.g. Lande and Arnold 1983; Kingsolver et al. 2002). As a result, the estimated selection gradients (the partial regression coefficients) reflect selection on a trait through a single episode of selection, rather than selection over multiple episodes or over a cohort's lifespan, as needed for evolutionary prediction. Focusing on this limitation, Arnold and Wade (1984a) considered partitioning the overall selection gradient into parts attributable to distinct episodes of selection, and Arnold and Wade (1984b) illustrated the approach with examples. Wade and Kalisz (1989) modified this approach to allow for change in phenotypic variance among selection episodes. Whereas these developments were intended to accommodate the multiple stages of selection, they do not directly account for the dependence of later components of fitness on ones expressed earlier, because they entail multiple separate analyses.

Further, Mitchell-Olds and Shaw (1987), among others, have noted that statistical testing of the selection gradients is often compromised by the failure of the analysis to satisfy the assumption of normality of the fitness measure, given the predictors. This concern applies to McGraw and Caswell's (1996) approach, which integrates observations from the full life-history. To address this problem for the case of dichotomous fitness outcomes, such as survival, Janzen and Stern (1998) recommended the use of logistic regression for testing selection on traits and showed how the resulting estimates could be transformed to obtain selection gradients. To allow for shapes of the fitness function more general than quadratic, Schluter (1988) and Schluter and Nychka (1994) suggested estimation of the relationship between fitness and traits as a cubic spline, but this also requires a parametric 
error distribution, whether normal, binomial, or Poisson.

Unconditional aster analysis estimates the relationship between overall fitness and traits directly in a single, unified analysis. We illustrate this use of aster with a reanalysis of Etterson's (2004) study of phenotypic selection on three traits in three populations of the annual legume, Chamaecrista fasciculata, reciprocally transplanted into three sites. The three traits, measured when the plants were 8-9 weeks old, are leaf number (LN, log transformed), leaf thickness (measured as specific leaf area, SLA, the ratio of a leaf's area to its dry weight, log transformed) and reproductive stage (RS, scored in 6 categories, increasing values denote greater reproductive advancement). Here, for simplicity, we consider a subset of the data for the three populations grown in the Minnesota site, comprising records on 2235 individuals.

In this experiment, individuals were planted as seedlings, and fitness was assessed as 1) survival to flowering, 2) flowering, given that the plant survived, 3) the number of fruits a plant produced, and 4) the number of seeds per fruit in a sample of three fruits, the last two contingent on the plant having flowered. For simplicity, we collapsed survival, flowering and fruiting to a single component of fitness, modeled as Bernoulli (reprod). Plants that produced fruit were assigned 1 for reprod, and those that didn't, regardless of the reason, 0. Consequently, overall fitness was modeled jointly as reproduction, number of fruits, and number of seeds in 3 fruits, (termed reprod, fruit, and seed, Fig. 1C). Preliminary analyses assessed the fit of truncated Poisson and truncated negative binomial distributions to the data for both fecundity components; on this basis the latter distribution was used for fruit and seed. In addition to the traits of interest, the model included as fixed effects the spatial blocks in which individuals were planted.

To illustrate phenotypic selection analysis most straightforwardly, we begin by analyzing two of the fitness components, reprod and fruit with graph Fig. 1D, in relation 
to the traits LN, SLA, and RS. We use an unconditional aster model, in which overall fitness is the number of fruits an individual seedling produced. This model detected strong dependence of fitness on all three traits such that selection is toward more leaves $\left(P<10^{-6}\right)$, thinner leaves $(P=0.006)$ and earlier $\left(P<10^{-6}\right)$ reproductive stage.

We detected highly significant negative curvature for LN and SLA suggestive of stabilizing selection, $\left(P<10^{-6}\right)$; because RS is categorical, we did not consider models quadratic in it. The plot of the fitness function together with the observed phenotypes (Fig. 3, solid contours) reveals that the fitness optimum lies very near the edge of the distribution of leaf number.

\section{EDITOR: PLACE FIGURE 3 HERE.}

Thus, for this trait, despite significant negative curvature, selection against both extremes of the standing variation in the trait (i.e. stabilizing selection) is not observed. The aster analysis fits the data well, as reflected by the scatter plots of Pearson residuals which show very little trend and only a few extreme outliers for fruit number (Fig. 4A).

\section{EDITOR: PLACE FIGURE 4 HERE.}

The assumptions for the aster model appear satisfied, and the estimated fitness surface is both biologically plausible and fits the data well. These points reinforce our confidence in the aster model $P$-values and estimated fitness surface.

We compare the result from aster modeling with that obtained by the approach of (Lande and Arnold 1983), which has become standard, ordinary least squares regression (OLS) of fruit count on traits. The bivariate fitness function inferred via OLS has positive curvature for LN, suggesting disruptive selection. This contrasts with the negative curvature obtained by aster. Fig. 3 reveals why OLS is misled. The fitness surface fitted by aster 
(solid contours) has a peak on the right side (large LN) and is fairly flat on the left

(small LN). The quadratic approximation (dotted contours) cannot have flat regions; its best approximation is a saddle. Further, a quadratic function cannot have both a saddle and a peak; thus OLS misses the peak. Another problematic feature of the quadratic approximation is that it goes negative. Thus, the main problem with OLS is the bias due to using a quadratic approximation to a highly non-quadratic surface. The aster model is also quadratic, but it is quadratic on the canonical parameter scale. The corresponding fitness estimates, which are mean value parameters, are necessarily positive.

The nominal $P$-values produced by OLS regression indicate that the positive curvature of the quadratic approximation in the LN direction is statistically significant $\left(P<10^{-6}\right)$, but the homoscedasticity and normality assumptions required for OLS regression to give meaningful $P$-values are seriously violated (Fig. 4B). Such violations of assumptions for an OLS regression analysis are expected, given that $3 \%$ of plants have fitness of zero and that the distributions of numbers of fruits per plant is heavily skewed. These violations make the nominal $P$-value from the OLS invalid.

We extend the above phenotypic selection analysis to include the additional fitness component, seed, using the graph Fig. 1C. In this case, fitness is no longer a canonical statistic, i.e. there is no linear combination of the variables corresponding to fitness. The two fecundity components, fruit and seed, are modeled as separately dependent on reprod. This analysis detected dependence of fruit on LN $\left(P<10^{-6}\right)$ and on SLA $(P=0.046)$ and of seed on both $\operatorname{LN}\left(P<10^{-6}\right)$ and $\operatorname{RS}\left(P<10^{-6}\right)$. It also found significant curvature in the relationship between fruit and both $\operatorname{LN}\left(P<10^{-6}\right)$ and SLA $(P=0.035)$ and between seed and $\operatorname{LN}(P=0.0008)$. Here again, we did not attempt to fit quadratic dependence on RS. Use of an unconditional aster model in these analyses yields an estimate of the relationship between each fitness component and each trait that takes 
into account pre-reproductive mortality.

The above analysis does not, however, satisfy the goal of evaluating the relationship between overall fitness and the traits; because fitness is not a canonical statistic, its expectation is not produced directly by aster methods. However, it can be approximated by averaging simulations. In detail, we use the estimated parameter values to simulate fitness records for individuals representing each trait combination, and from these, we calculate fruit $*$ seed / 3 . The average over the simulated fitness values for each trait combination approximates its expected fitness. The resulting fitness surface (Fig. 5, solid contours) resembles that estimated using only fruit as the fecundity measure, though it provides more compelling evidence of true stabilizing selection on LN. The best quadratic approximation fitted by OLS has a saddle also in this case.

\section{EDITOR: PLACE FIGURE 5 HERE.}

There are two alternative data structures in which a simple aster analysis, needing no simulation, would directly analyze fitness. The graph $1 \rightarrow$ reprod $\rightarrow$ fruit $\rightarrow$ seed would be appropriate if all seeds (from all fruits) had been counted for each individual. Then, in an unconditional aster model, fitness would be seed, a canonical statistic, and the analysis would automatically take the contribution of reprod and fruit to fitness into account. However, it is often impractical, as in this case, to count all seeds. Subsampling is a common practice in studies of animals (e.g. Howard 1979), as well as plants. An alternative to exhaustive enumeration that facilitates aster analysis is to obtain for each individual the seed count for a random number of fruits, corresponding to the graph $1 \rightarrow$ reprod $\rightarrow$ fruit $\rightarrow$ samp $\rightarrow$ seed, where samp is the number of fruits sampled for the individual, a binomial(fruit, $p$ ) random variable, where $p$ is fixed and known (the fraction of fruit sampled). In this sampling scheme, fitness would be proportional to seed, a canonical statistic, and aster analysis would be simplified. 
For our actual data, it might seem natural to use the product of fruit count

420

and number of seeds per fruit as one variable in an aster analysis, so the graph is $1 \rightarrow$ reprod $\rightarrow$ fruit $*$ seed / 3 but this would not be valid, because this product is not distributed according to an exponential family. Alternatively, it might seem natural to use the preceding graph with samp replaced by the constant 3 , but this is also invalid because the constant 3 is not distributed according to an exponential family. Thus, the structure of this aster model precluded inference of overall selection via a simple aster analysis. Nevertheless, simulation yielded the expected fitness surface.

In this section, we have illustrated how aster can conduct phenotypic selection analysis on complete life-history records to yield more biologically interpretable estimates of the fitness surface. Moreover, even for analysis of an annual life-history, commonly considered relatively straightforward, aster greatly improves over OLS in its adherence to statistical assumptions and, accordingly, in the validity of the inferences. We have also shown that, even when the available data preclude modeling total reproductive output as sequentially dependent on all earlier expressed fitness components, aster estimates the parameters of a fitness model that can be used (with simulation, if necessary) to produce a statistically sound phenotypic selection analysis.

\section{Discussion}

Both the numerical and genetic dynamics of a population depend fundamentally on individuals' contributions of offspring, their fitness. Extensive theoretical work (e.g. Fisher 1930; Charlesworth 1980) has formalized and extended this insight of Darwin, yet statistical challenges have continued to compromise the empirical evaluation of fitness. The aster approach addresses these challenges and takes full advantage of available data to yield comprehensive assessments of fitness that are as precise as possible. The 
precision of aster modeling not only offers statistical power for tests of hypotheses; it also

444 promotes quantitative comparison of fitnesses. Most important, as a general framework for analyzing life-history data, aster can address questions that arise in diverse evolutionary and ecological contexts. The examples presented here illustrate the breadth of aster's applicability, including estimation of population growth rate, comparison of mean fitness among groups, and inference of phenotypic selection. Even beyond analysis of life-history data, aster modeling is appropriate for any set of responses in which there are dependencies analogous to those characteristic of life-histories. In an experimental study of foraging behavior, for example, individual subjects may forage in a given interval or not and, given that they forage, may take varying numbers of prey. In a medical context, aster can expand on survival analysis by incorporating measures of patients' well-being in evaluating the relative benefits of different procedures. We emphasize that aster obviates the common practice of multi-step analysis, which cannot provide valid statistical tests or sound estimates of sampling error. A single aster model can encompass the real complexities not only of life-histories, but also of discrete and continuous predictors, and thus provide a full analysis to yield direct inferences about fitness and population growth.

Lifetime fitness rarely, if ever, conforms to any distribution amenable to parametric statistical analysis less complex than aster analysis. This pathology of fitness distributions has plagued empirical studies of fitness. Resampling approaches are sometimes used, but this is not a general solution, because valid resampling schemes are not generally available for complex data structures. Moreover, resampling methods sacrifice statistical precision relative to parametric analysis. As an alternative, transformations are often attempted, but the prevalence of mortality before reproduction typically results in fitness distributions with many individuals at zero, such that no transformation produces a well known distribution. Moreover, even if such a transformation could be found, analyses of fitness on an alternative scale can mislead (Stanton and Thiede 2005). Aster addresses these problems by directly 
modeling each distinct component of fitness with a suitable parametric distribution and

470 accounting for the dependence of each fitness component on those expressed earlier. As a consequence, it models the sampling variation appropriately and yields results on the biologically natural scale of expected number of individuals produced per individual. Even when life-history records are available for only a portion of the life-span of a cohort, as in our Example 2, joint analysis via an unconditional aster model provides comparisons based on the most comprehensive fitness records at hand.

Studies of variation in fitness often focus on a single component of fitness (e.g. Arnold and Lande 1983). These are less subject to distributional problems and can yield insight into the nature of fitness variation during a particular episode of selection. However, the resulting understanding of fitness and its variation is fragmentary and can be misleading when the relationship between components of fitness, on the one hand, and traits or genotypes, on the other, varies over the lifespan (Prout 1971). Whereas Arnold and Wade (1984a) proposed an approach to evaluate phenotypic selection over multiple episodes (modified by Wade and Kalisz 1989), this approach uses separate analyses of each episode, ignoring the dependence structure of fitness components. Consequently, the sampling variance of the resulting estimates of selection cannot readily be determined.

Our first example illustrates use of aster to infer population growth rate. Lenski and Service (1982) first noted the importance of sound statistical modeling for population growth. Our use of aster in this context builds on their work by employing parametric models for each life-history event. The resulting estimate of growth rate is similar to that obtained by Lenski and Service's (1982) method using the jackknife, as are the confidence intervals from the two approaches. The key point is that aster analysis provides a sound parametric basis for inferences about population growth even for data structures that are not suited to resampling. Though we have emphasized the utility of unconditional aster 
models for inferences about lifetime fitness, conditional aster models also offer the capability of obtaining estimates of expected values of a life-history component, conditional on earlier life-history status (e.g. fecundity at a given age, given survival to that age), as we have done in this example.

In the example of Lenski and Service (1982), complete records are available for each individual in a single cohort, so the life table can be based on age. Life histories are often tabulated in relation to size or stage categories instead of age, because size or stage often predicts survival and fecundity better than age does (Caswell 2001, chap. 2); this is especially useful when ages of individuals are unknown, for example, in censuses of populations in nature, as in the examples of Alvarez-Buylla and Slatkin (1994). Though our examples include only cases based on age, aster analysis is also suitable for analyzing life-history data according to stage or size.

Our second example demonstrates the use of unconditional aster models to estimate and compare mean fitness for groups produced by different mating schemes and, thus, differing in genetic composition. This analysis reveals that the remnant populations of E. angustifolia are subject to severe inbreeding depression of at least $70 \%$ overall, when extrapolated linearly to inbreeding arising from one generation of selfing. In a similar application of aster, Geyer et al. (2007) have analyzed survival and annual production of flower heads jointly for samples of these remnant populations grown in the common field. This analysis revealed greater than twofold differences in mean fitness among remnants $(P<0.01)$. In the case we provide here, we further demonstrate how the likelihood framework of aster permits straightforward tests of several hypotheses. We show that the significant early disadvantage in size and survival of inbred plants does not adequately account for the fitness differences at the end of the period of observation. Rather, inbreeding depression in growth and survival exacerbates the fitness disparity beyond the first three 
months. Thus, in addition to providing statistically rigorous comparisons of overall fitness among groups, aster yields insights into the timing of fitness effects.

Aster readily extends further to accomplish phenotypic selection analysis, by establishing the relationship between individuals' overall demographic-genetic contribution to the next generation and the traits they express. For this, the aster model includes the traits under consideration as predictors of cumulative fitness; inference of quadratic and correlational selection is also straightforward. Our Example 3 shows aster's estimation of the fitness surface when fitness is a linear function of the components of fitness and also demonstrates how to obtain such an estimate even when it is not. Van Tienderen (2000) presented a method with a similar goal, but it does not take into account the dependence relationships of the fitness components and is subject to the usual distributional problems (e.g. Coulson et al. 2003). Further, it cannot validly represent the statistical uncertainty of inferred parameters because it involves separate analyses to estimate selection gradients for each fitness component. In our examples 2 and 3 and the example in Geyer et al. (2007), use of an unconditional model was essential to obtain results that are interpretable as comparisons of overall fitness. In our examples $P$-values and confidence intervals reported are asymptotic, but we do not need to rely on asymptotic normality of MLE because the parametric bootstrap is easily done with the aster software and does not require this assumption.

The centrality of fitness to many evolutionary and ecological questions demands a statistical approach that rigorously models the inevitable, complex dependencies of life-history data. Our examples provide only a glimpse of the range of possible uses of aster models. Conceivably, all the issues in all our examples and more besides, could arise in a single analysis, as could more extensive dependence. The aster approach addresses these challenges. Its versatility suits it to answer the full breadth of questions that life-history 
544

546

548

data can address. Aster can play a key role in linking ecological and evolutionary study of populations.

RGS and CJG cordially thank Janis Antonovics for his encouragement when we began work on the basic idea about 1980 and for funding its development then, as well as 548 for his enthusiasm about its eventual realization. Computational challenges stymied the initial efforts, and other work intervened until the richness of life-history data from recent experiments stimulated us to revisit the idea. For very helpful suggestions for clarifying the manuscript, we thank J. Antonovics, K. Mercer, M. Price, P.D. Taylor, J. Travis, N. Waser and an anonymous reviewer. Examples 2 and 3 are drawn from research funded by NSF (DMS-0083468, DEB-0545072, DEB-0544970) and EPA STAR graduate student fellowship (U 914758-01-2) respectively, as well as the University of Minnesota Center for Community Genetics. 


\section{REFERENCES}

Alvarez-Buylla, E. R., and M. Slatkin. 1994. Finding confidence limits on population growth rates: three real examples revised. Ecology 75:255-260.

Antonovics, J., and N. C. Ellstrand. 1984. Experimental studies of the evolutionary significance of sexual reproduction. I. A test of the frequency-dependent selection hypothesis. Evolution 38:103-115.

Arnold, S. J., and M. J. Wade. 1984a. On the measurement of natural and sexual selection: theory. Evolution 38:709-719.

Arnold, S. J., and M. J. Wade. 1984b. On the measurement of natural and sexual selection: applications. Evolution 38:720-734.

Barndorff-Nielsen, O. E. 1978. Information and exponential families. John Wiley, Chichester.

Belaoussoff, S., and J. S. Shore. 1995. Floral correlates and fitness consequences of mating-system variation in Turnera ulmifolia. Evolution 49:545-556

Caswell, H. 2001. Matrix population models: construction, analysis, and interpretation, 2nd ed. Sinauer Associates, Sunderland, Mass.

Charlesworth, B. 1980. Evolution in age-structured populations. Cambridge University Press, Cambridge.

Cheng, S., D. Wang, and E. P. Smith. 2000. Adjusting for mortality effects in chronic toxicity testing: mixture model approach. Environmental Toxicology and Chemistry 19:204-209. 
Coulson, T., L. E. B. Kruuk, F. Tevacchia, J. M. Pemberton, and T. H. Clutton-Brock. 2003. Estimating selection on neonatal traits in red deer using elasticity path analysis. Evolution 57:2879-2892.

Dagne, G. A. 2004. Hierarchical Bayesian analysis of correlated zero-inflated count data. Biometrical Journal 46:653-663.

Etterson, R. R. (2004) Evolutionary potential of Chamaecrista fasciculata in relation to climate change. I. Clinal patterns of selection along an environmental gradient in the great plains. Evolution 58:1446-1458.

Fisher, R. A. 1930. The genetical theory of natural selection. Clarendon Press, Oxford. [Reprinted Dover]

Galloway, L. F., and J. R. Etterson. 2007. Inbreeding depression in an autotetraploid herb: a three cohort field study New Phytologist 173:383-392.

Geyer, C. J., S. Wagenius, and R. G. Shaw. 2007. Aster models for life history analysis. Biometrika 94:415-426.

Howard, R. D. 1979. Estimating reproductive success in natural populations. American Naturalist 114:221-231.

Husband, B. C., and D. W. Schemske. 1996. Evolution of the magnitude and timing of inbreeding depression in plants. Evolution 50:54-70.

Janzen, F. J., and H. S. Stern. 1998. Logistic regression for empirical studies of multivariate selection. Evolution 52:1564-1571.

Kingsolver, J. G., H. E. Hoekstra, J. M. Hoekstra, D. Berrigan, S. N. Vignieri, C. E. Hill, A. Hoang, P. Gilbert, and P. Beerli. 2001. The strength of phenotypic selection in natural populations. The American Naturalist 157:245-261. 
Lande, R., and S. J. Arnold. 1983. The measurement of selection on correlated characters. Evolution 37:1210-1226.

Lehmann, E. L., and G. Casella. 1998. Theory of Point Estimation, 2nd ed. Springer-Verlag, New York.

Lenski, R. E., and P. M. Service. 1982. The statistical analysis of population growth rates calculated from schedules of survivorship and fecundity. Ecology 63:655-662.

McCullagh, P., and J. A. Nelder. (1989). Generalized Linear Models, 2nd ed. Chapman \& Hall, London.

McGraw, J. B., and H. Caswell. 1996. Estimation of individual fitness from life-history data. American Naturalist 147:47-64.

Martin, T. G., B. A. Wintle, J. R. Rhodes, P. M. Kuhnert, S. A. Field, S. J. Low-Choy, A. J. Tyre, and H. P. Possingham. 2005. Zero tolerance ecology: improving ecological inference by modelling the source of zero observations. Ecology Letters 8:1235-1246.

Mitchell-Olds, T., and R. G. Shaw. 1987. Regression analysis of natural selection: statistical inference and biological interpretation. Evolution 41:1149-1161.

Mood, A. M., F. A. Graybill, and D. C. Boes. 1974. Introduction to the theory of statistics, 3rd ed. McGraw-Hill, New York.

Morris, W. F., and D. F. Doak. 2002. Quantitative conservation biology: theory and practice of population viability analysis. Sinauer Associates, Sunderland, Mass.

Prout T. 1971. The relation between fitness components and population prediction in Drosophila. I: the estimation of fitness components. Genetics 68:127-149. 
R Development Core Team. 2006. R: A language and environment for statistical computing. R Foundation for Statistical Computing, Vienna. http://www.r-project.org.

Schluter, D. 1988. Estimating the form of natural selection on a quantitative trait. Evolution 42:849-861.

Schluter, D., and D. Nychka. 1994. Exploring fitness surfaces. American Naturalist 143:597-616.

Shaw, R. G. 1986. Response to density in a wild population of the perennial herb Salvia lyrata: variation among families. Evolution 40:492-505.

Shaw, R. G., C. J. Geyer, S. Wagenius, H. H. Hangelbroek, and J. R. Etterson. 2007a. Supporting data analysis for "Unifying life history analysis for inference of fitness and population growth". Technical Report No. 658, School of Statistics, University of Minnesota. http://www.stat.umn.edu/geyer/aster

Shaw, R. G., C. J. Geyer, S. Wagenius, H. H. Hangelbroek, and J. R. Etterson. 2007b. More supporting data analysis for "Unifying life history analysis for inference of fitness and population growth". Technical Report No. 661, School of Statistics, University of Minnesota. http://www.stat.umn.edu/geyer/aster

Stanton, M. L., and D. A. Thiede. 2005. Statistical convenience vs. biological insight: consequences of data transformation for the analysis of fitness variation in heterogeneous environments. New Phytologist 166:319-337.

van Tienderen, P. H. 2000. Elasticities and the link between demographic and evolutionary dynamics. Ecology 81:666-679.

Wade, M. J. and S. Kalisz. 1989. The additive partitioning of selection gradients. Evolution 43:1567-1569. 
${ }_{644}$ Wagenius, S. 2000. Performance of a prairie mating system in fragmented habitat: self-incompatibility and limited pollen dispersal in Echinacea angustifolia. Ph.D. dissertation. University of Minnesota.

This manuscript was prepared with the AAS LATEX macros v5.2. 


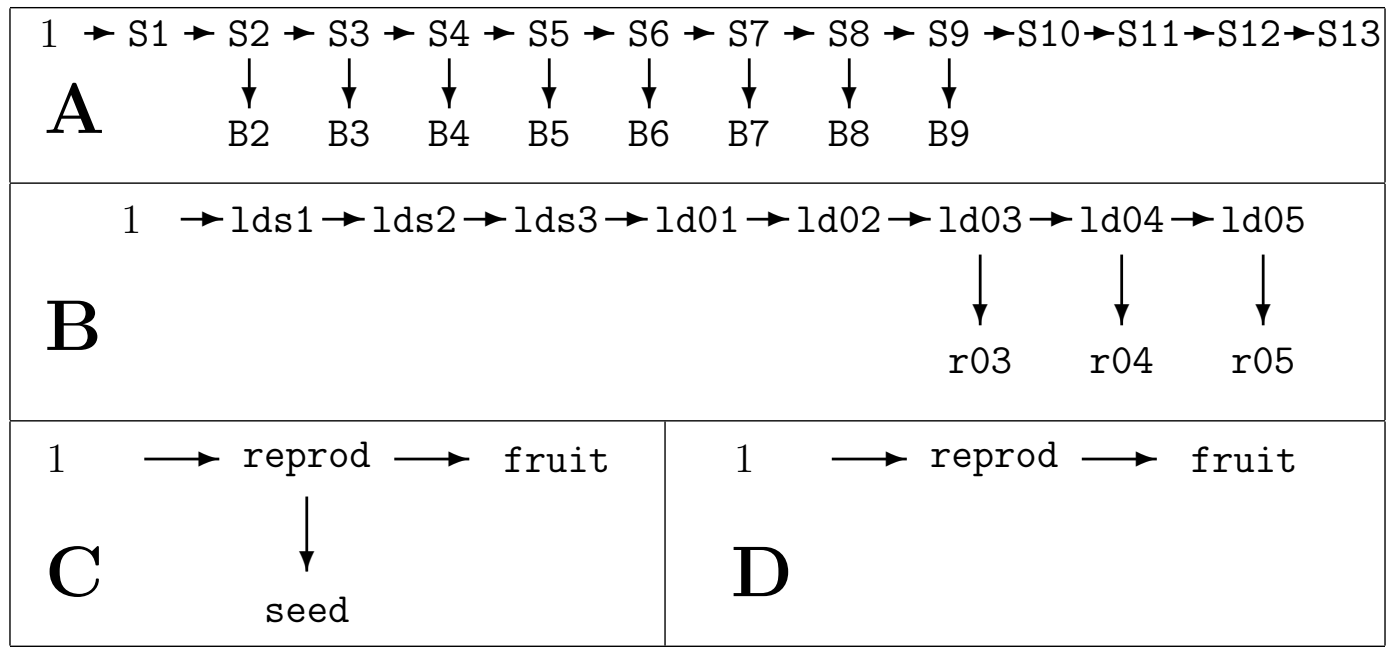

Fig. 1.- Graphical models for our three empirical examples. Each node of a graph is associated with a variable, root nodes with the constant variable 1, indicating presence of an individual at the outset. Arrows lead from one life history component to another that immediately depends on it (from predecessor node to successor node of the graph). If a predecessor variable is nonzero, then a particular conditional distribution of the successor variable is assumed. If a predecessor variable is zero for a given individual, for example due to mortality, then its successor variables are also zero. A: Example 1: Uroleucon rudbeckiae, an aphid. An individual's fitness is determined by its survival to each age, si, modeled as (conditionally) Bernoulli and the number of young it produces at each age, Bi modeled as (conditionally) zero-truncated Poisson B: Example 2: Echinacea angustifolia, a perennial plant. Fitness comprises juvenile survival at three times up to transplanting into the field (ldsi) and subsequent survival through five years (1d0i), as well as the plant's number of rosettes $(\mathrm{r} 0 \mathrm{i})$ in three years. The survival variables are modeled as (conditionally) Bernoulli (zero indicates mortality, one indicates survival), and $r 0 i$ is (conditionally) zero-truncated Poisson (i.e. a Poisson random variable conditioned on being greater than 0). C and D: Example 3: Chamaecrista fasciculata, an annual plant. Success or failure of reproduction (here, including survival to reproduction) is given by reprod, modeled as Bernoulli (zero indicates no seeds, one indicates survival to reproduction). Given that a plant reproduces, the components of its fecundity are its number of fruits (fruit) and, in $\mathrm{C}$ its and number of seeds per fruit in a sample of three fruits (seed). Each are modeled as negative binomial, respectively, two-truncated and zero-truncated. 

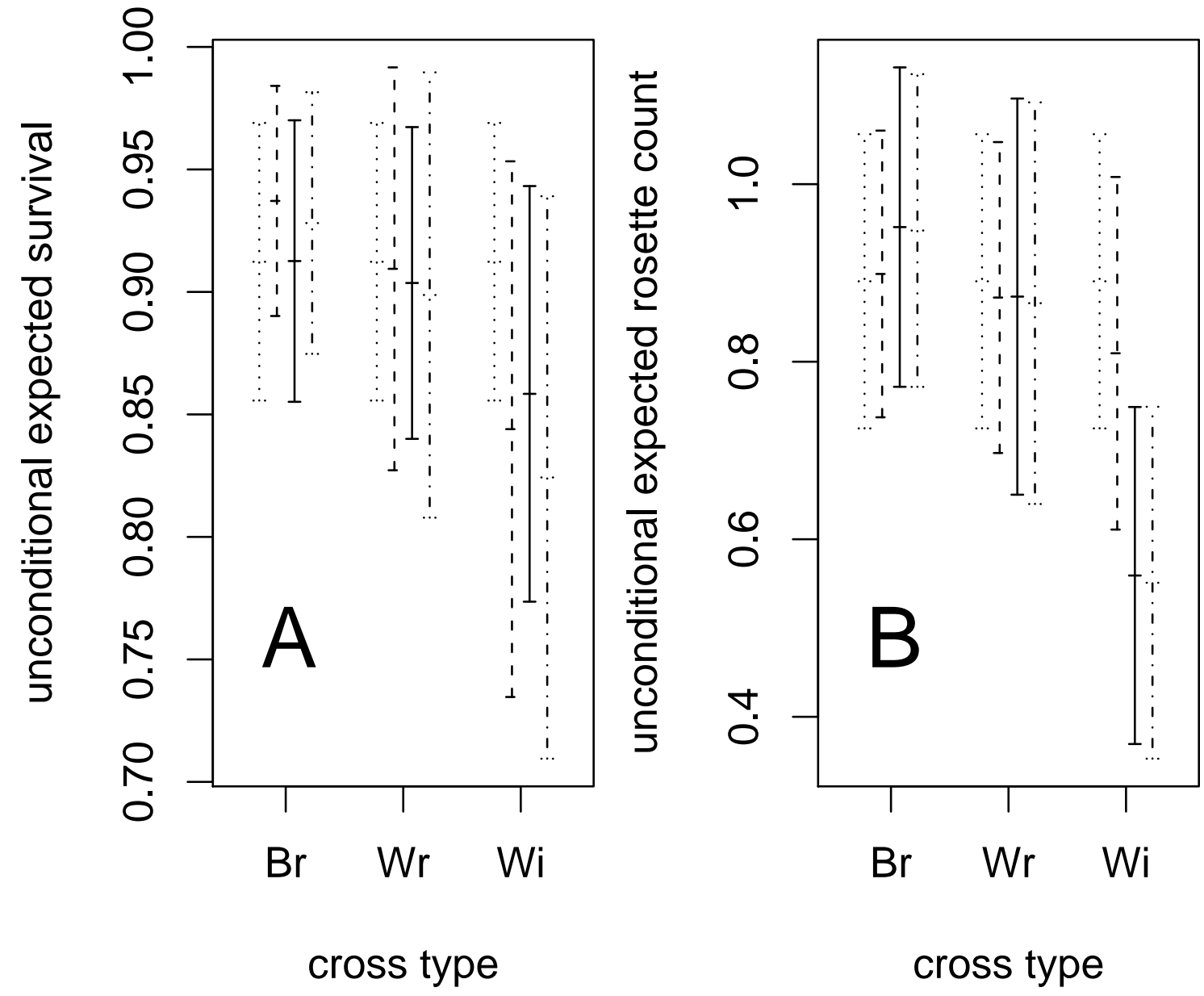

Fig. 2.- Predicted values and 95\% confidence intervals for the unconditional mean value parameter for (A) survival up to transplanting and (B) rosette count in the last year recorded (i.e. overall fitness over the study period) for a "typical" individual for each cross type. The experimentally imposed crossing treatments are Br, between remnant populations; Wr, within remnant populations; and $\mathrm{Wi}$, inbred within remnants (i.e. between sibs). Lines indicate model: dotted, "sub" model; dashed, "chamber" model; solid, "field" model; dotdash, "super" model. 


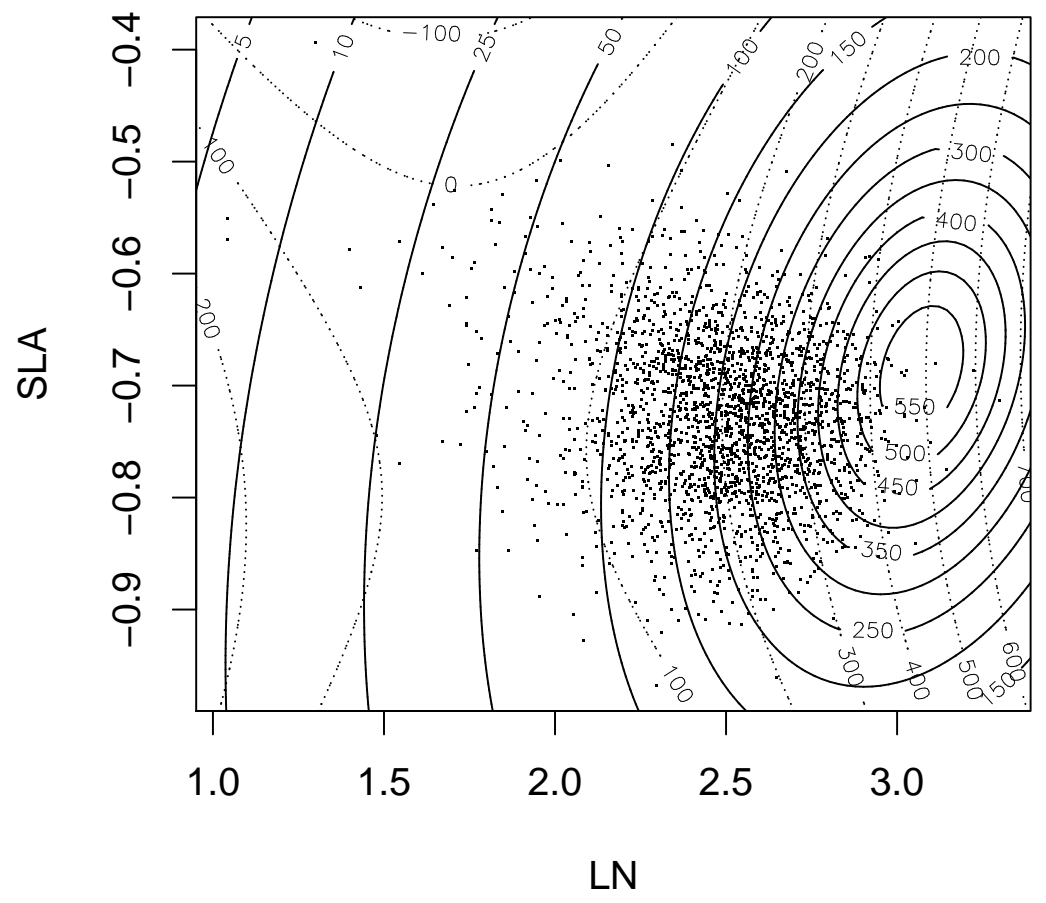

Fig. 3.- Scatterplot of SLA (specific leaf area, ln transformed) versus LN (leaf number, ln transformed) with contours of the fitness function (expected fruit count) estimated by aster (solid) and its quadratic approximation via Ordinary Least Squares (dotted). Cf. Fig. 5. 

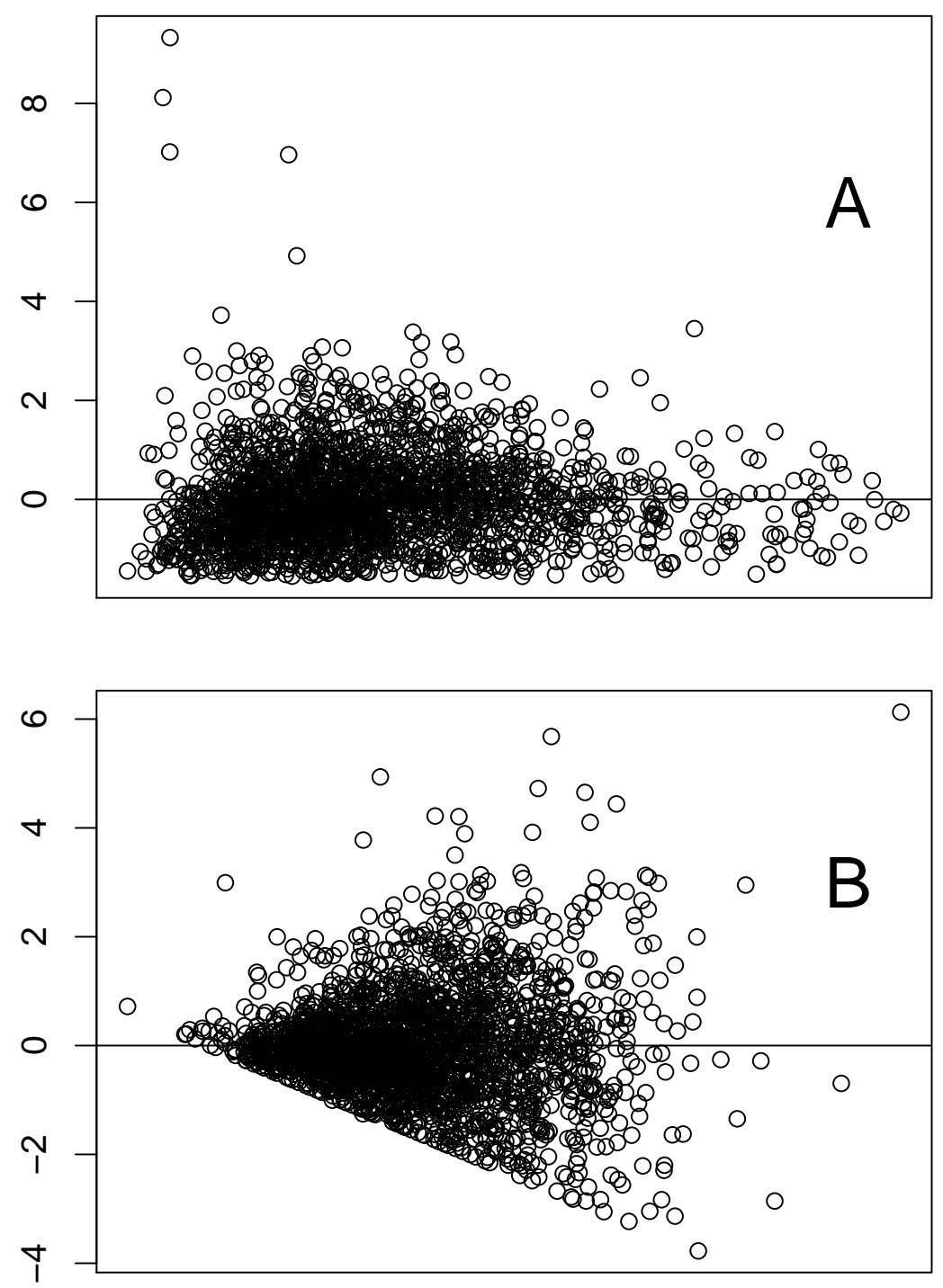

Fig. 4.- Residual plots from phenotypic selection analyses for Chamaecrista fasciculata. A. Pearson residuals for fruit count conditional on reproduction plotted against values fitted from the aster model quadratic in leaf number (LN) and specific leaf area (SLA) and also containing reproductive stage $(\mathrm{RS})$ and spatial block. B. Similar except standardized residuals fitted by ordinary least squares (same response and predictors as in A). 


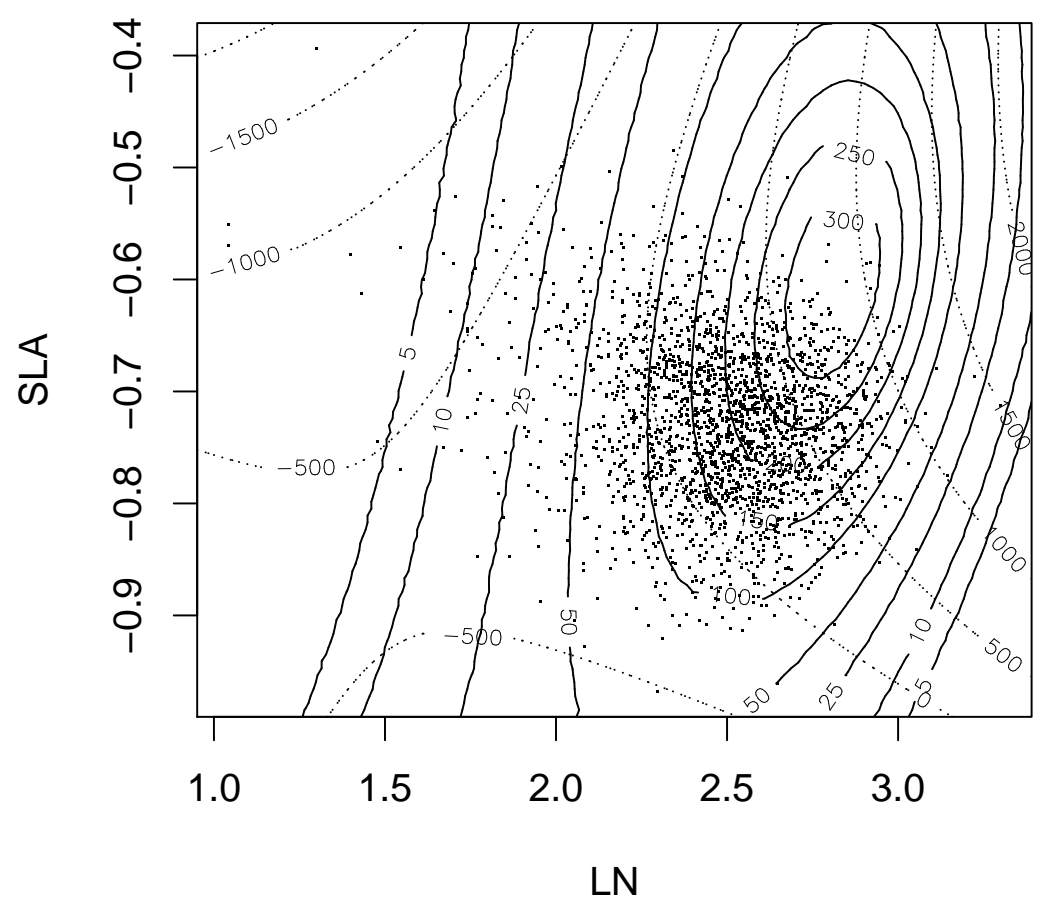

Fig. 5.- Scatterplot of SLA (specific leaf area, ln transformed) versus LN (leaf number, ln transformed) with contours of the fitness function (expectation of fruit times seed divided by three) estimated by aster (solid) and the quadratic approximation via Ordinary Least Squares (dotted). Cf. Fig. 3. 